\title{
Maternal and Fetal Outcomes of COVID-19 Pregnant Women Followed Up at a Tertiary Care Unit: A Descriptive Study
}

\author{
Madye A. Ngo Dingom ${ }^{1}$, Eugène Sobngwi ${ }^{2,3}$, Félix Essiben ${ }^{1,3}$, Antoinette N. Assiga ${ }^{2}$, \\ Yves F. Wasnyo ${ }^{2}$, Anicet Ngate ${ }^{3}$, Jean C. Katte ${ }^{4}$, Junie Y. Ngaha1, Philemon Nsem', \\ Charles E. Sone', Brigitte Wandji', Gilles T. Libend', Jean J. Bissemou ${ }^{1}$, Filbert E. Eko ${ }^{1,5}$, \\ Florent Y. Fouelifack ${ }^{1,5}$, Glwadys Ngono ${ }^{3}$, Jeanne Fouédjio ${ }^{1,3}$, Rebecca Tonye ${ }^{1}$, \\ Pierre Ongolo-Zogo ${ }^{2,3}$, Pierre J. Fouda ${ }^{2,3}$, Robinson E. Mbu ${ }^{1,3}$ \\ ${ }^{1}$ Obstetrics and gynecology Unit, Yaounde Central Hospital, Yaounde, Cameroon \\ ${ }^{2}$ COVID 19 Unit, Yaounde Central Hospital, Yaounde, Cameroon \\ ${ }^{3}$ Department of Medicine and Biomedical Sciences, University of Yaounde I, Yaounde, Cameroon \\ ${ }^{4}$ Department of Public Health, University of Exeter, Exeter, UK \\ ${ }^{5}$ Department of Obstetrics and Gynecology, Higher Institute of Medical Technology of Nkolondom, Yaoundé, Cameroon \\ Email: ${ }^{*}$ ingom20022003@yahoo.fr
}

\begin{abstract}
How to cite this paper: Dingom, M.A.N., Sobngwi, E., Essiben, F., Assiga, A.N., Wasnyo, Y.F., Ngate, A., Katte, J.C., Ngaha, J.Y., Nsem, P., Sone, C.E., Wandji, B., Libend, G.T., Bissemou, J.J., Eko, F.E., Fouelifack, F.Y., Ngono, G., Fouédjio, J., Tonye, R., Ongolo-Zogo, P., Fouda, P.J. and Mbu, R.E. (2020) Maternal and Fetal Outcomes of COVID-19 Pregnant Women Followed Up at a Tertiary Care Unit: A Descriptive Study. Open Journal of Obstetrics and Gynecology, 10, 1482-1491.

https://doi.org/10.4236/ojog.2020.10100135
\end{abstract}

Received: September 7, 2020

Accepted: October 25, 2020

Published: October 28, 2020

Copyright $\odot 2020$ by author(s) and Scientific Research Publishing Inc. This work is licensed under the Creative Commons Attribution International License (CC BY 4.0).

http://creativecommons.org/licenses/by/4.0/ (c) (i) Open Access

\begin{abstract}
Background: The SARS-CoV-2 and associated corona virus disease COVID-19 have been declared a pandemic having a poor prognosis among individuals with debilitating conditions and those who are immune-compromised. Current evidence is however limited on maternal and fetal transmission and pregnancy outcomes. We aimed to describe the pattern of SARS-CoV-2 infection and outcomes in a group of pregnant women followed up at a tertiary care unit in Cameroon. Methods: This was an observational study conducted over a period of 3 months (April 1 to June 30, 2020) at the Yaounde Central Hospital. All pregnant women who were tested positive for SARS-CoV-2 and who provided a signed written informed consent were included in the study. Results: Out of 83 pregnant women who presented with symptoms suspicious of COVID-19, 25 were tested positive. The median age of these women was $31(27-35)$ years. A total of $76 \%$ consulted within 6 days of onset of symptoms and $68 \%$ had a gestational age greater than 28 weeks. The most common presenting complaint was fever (88\%). All 25 pregnant women who were tested positive for COVID-19 were followed up with 9/25 deliveries registered. There were two intra-uterine fetal deaths and seven live births. All the live birth babies were tested negative on Real Time-Polymerase Chain Reaction (RT-PCR) testing which was performed after birth. Conclusion: Deliverance of live and SARS-CoV-2 negative babies from COVID-19 pregnant women is possible.
\end{abstract}




\section{Keywords}

COVID-19, Pregnancy, Mortality, Outcomes

\section{Background}

In December 2019, the first cases of pneumonia due to a new coronavirus, SARS-CoV-2 were described in China [1]. In early March 2020, the disease, now known as COVID-19, was declared a pandemic by the World Health Organisation (WHO). To date, the world has nearly 13,616,593 cases and approximately 585,727 deaths [2] among which 16,057 cases and 369 deaths have been recorded in Cameroon as of July 17, 2020 [2]. COVID-19 is now known to portray a vast array of clinical manifestations ranging from asymptomatic individuals to overt respiratory distress [3]. The disease is thought to be severe in the elderly and patients with chronic debilitating conditions such as hypertension, heart failure and diabetes and other immune-compromised states.

Pregnancy is generally known to be an immune-compromised condition, so pregnant women may be at risk of severe COVID-19 given that back in 2003 and 2012 fatal forms of coronavirus pneumopathy were identified with SARS-CoV-1 and MERS-CoV infections in pregnancy [4] [5]. However, to date, the available data related to SARS-CoV-2 infection do not indicate a higher infection rate or increased risk of severity in pregnant women compared to the general population [6] [7]. There are few descriptions of maternal-fetal transmission of COVID-19 and their corresponding outcomes in the literature at the moment and none has otherwise been described in sub-Saharan Africa. We therefore aimed to describe the outcomes of maternal and fetal transmission of COIVD-19 in a group of women followed up at the Yaounde Central Hospital in Cameroon.

\section{Methods}

\subsection{Study Design and Setting}

This was a prospective observational study which lasted for 3 months (April 1 to June 30, 2020) at the Obstetric and Gynecological Unit of the Yaounde Central Hospital in Cameroon. This unit provides tertiary care to pregnant women and serves as a referral unit for over 40 district hospitals in the centre region of Cameroon. The unit is served by 14 physicians and 20 non-physician staff. Mask wearing was compulsory for all staff and patients in the unit with staff using additional prescribed protective gears.

\subsection{Study Participants}

All COVID-19 positive pregnant symptomatic women who were screened-diagnosed [via the Real-Time Polymerase Chain Technique (RT-PCR) or Rapid Diagnostic Test (RDT)] during the time of the study were included after a signed written in- 
formed consent was provided. All data provided by the participants were recorded in anonymised forms which were available only to the clinical and research team.

\subsection{Data Collection}

Data was collected from the participants' prenatal consultation and pregnancy monitoring record as well as their referral sheet (if they were referred for care with a positive COVID-19 test). Data was recorded onto a pre-structured data collection form designed for the study. We interviewed the participants or their accompanying family carer to complete their socio-demographic characteristics, medical and pregnancy history, presenting complaints, treatment received, and the pregnancy outcomes. A complete physical examination was performed by the attending physician or nurse and obstetric parameters were also recorded.

\subsection{Data Management}

Data was entered into a prepared Microsoft Excel 2010 Spreadsheet by a non-clinical staff. The data was then double-checked item by item to ensure accuracy and completeness. The data was later exported to SPSS version 23 for analysis. Data was expressed in frequency, percentage and median (interquartile range) were necessary.

\section{Results}

\subsection{Baseline Characteristics of the Study Participants}

Over the 3 months period, a total of 1197 pre-natal consultations were registered with 83 pregnant women tested for SARS-CoV-2 based on their presenting symptoms, of whom 25 had a positive COVID-19 test. Of the 25 pregnant women, 23 were PCR-confirmed cases while 2 were positive on a serologic test. Table 1 shows the baseline characteristics of the study participants. The majority (64\%) of women were aged between 25 to 35 years. Most patients came to consult within at least 6 days after the onset of initial symptoms (76\%), and more than half of them having a gestational age greater than 28 weeks of amenorrhea (68\%).

\subsection{Presenting Symptoms of COVID-19 Positive Pregnant Women}

Twenty-two patients $(88 \%)$ had fever $\left(\geq 39^{\circ} \mathrm{C}\right)$, eighteen (80\%) presented with cough. Nineteen (76\%) reported intense fatigue. Symptoms of respiratory involvement were seen as follows: Ten (40\%) had respiratory distress, eight (32\%) had a rhinorrhoea, 7 (28\%) had anosmia and six (24\%) had ageusia; Three patients $(8 \%)$ had diarrhoea. Ten $(40 \%)$ of our patients developed severe respiratory distress requiring admission to the intensive care unit. Figure 1 shows the presenting complaints. 
Table 1. Socio-demographic characteristics of the study population.

\begin{tabular}{|c|c|c|}
\hline Characteristics & Frequency $(\mathrm{n}=25)$ & Percentage (\%) \\
\hline \multicolumn{3}{|l|}{ Age, years } \\
\hline $15-24$ & 3 & 12.0 \\
\hline $25-35$ & 16 & 64.0 \\
\hline$>35$ & 6 & 24.0 \\
\hline \multicolumn{3}{|l|}{ Marital status } \\
\hline Single & 7 & 28.0 \\
\hline Married & 18 & 72.0 \\
\hline \multicolumn{3}{|c|}{ Onset of symptoms before consultation } \\
\hline $1-5$ days & 6 & 24.0 \\
\hline $6-10$ days & 11 & 44.0 \\
\hline$>10$ days & 8 & 32.0 \\
\hline \multicolumn{3}{|l|}{ Parity } \\
\hline Primiparous & 4 & 16.0 \\
\hline Multiparous & 21 & \\
\hline \multicolumn{3}{|c|}{ Gestational age of pregnancy (weeks) } \\
\hline$<14$ weeks & 2 & 8.0 \\
\hline $15-28$ weeks & 6 & 24.0 \\
\hline$>28$ weeks & 17 & 68.0 \\
\hline
\end{tabular}

Median age $(\mathrm{IQR})=31(27-35)$ years; $\operatorname{Min}-\mathrm{Max}=18-43$ years.

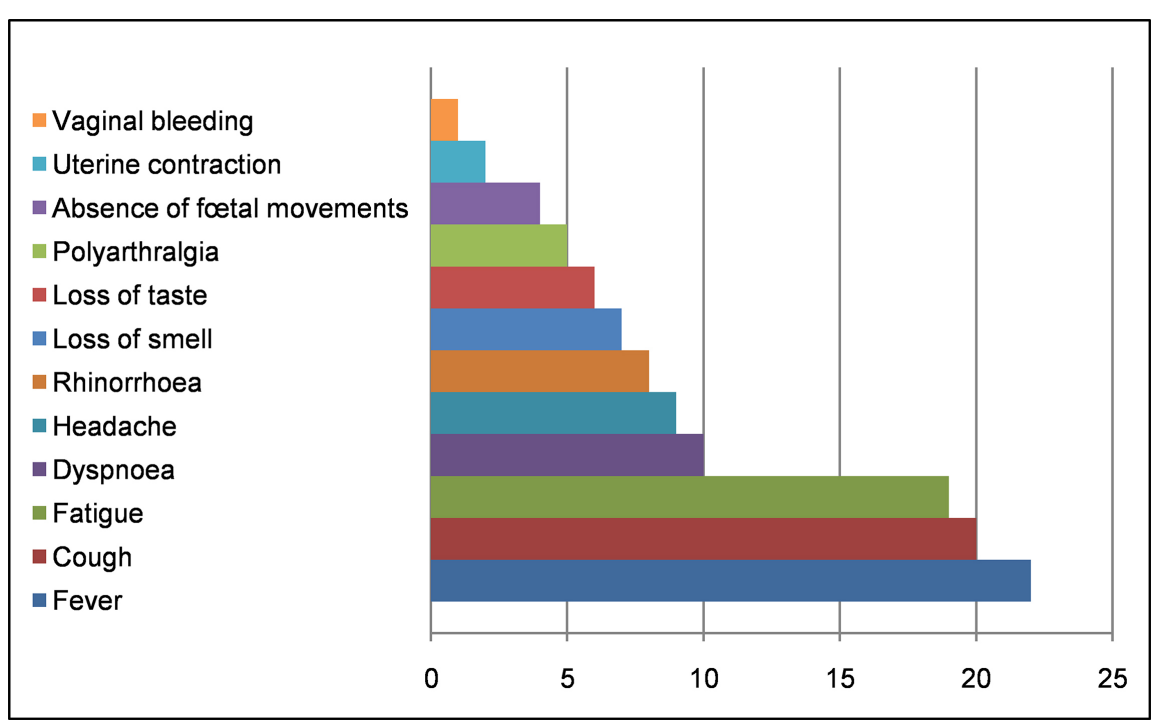

Figure 1. Presenting symptoms of the study participants presenting with COVID-19. 


\subsection{Treatment Received for COVID-19}

Antibiotic (96\%) and anti-parasitic (88\%) treatment had been initiated in the majority of patients. Ten (40\%) patients received oxygen therapy and seven (28\%) were treated with anti-coagulant therapy (see Table 2).

\subsection{Maternal and Fetal Outcomes and Prognosis}

Obstetrically, four (16\%) patients had their pregnancies terminated, two (8\%) had notified the occurrence of uterine contractions and one (4\%) had per vaginal bleeding (Figure 2). All the pregnant women were alive by the end of the study. Regarding the outcome of pregnancy, we noted that fourteen (56\%) women have a current pregnancy. Among the nine (36\%) women who gave birth, four (44.4\%) had a cesarean section. Acute fetal distress was the indication for cesarean section in three cases. Among the nine births, there were seven live births. All seven neonates were tested negative for COVID-19 via RT-PCR after birth (see Table 3).

Table 2. Treatment received during follow-up.

\begin{tabular}{ccc}
\hline Treatment type & Frequency $(\mathrm{n}=25)$ & Percentage (\%) \\
\hline Azithromycin & 24 & 96.0 \\
Chloroquine and other anti-malarial agent & 22 & 88.0 \\
Vitamin C & 18 & 72.0 \\
Zinc 10mg tablet & 17 & 68.0 \\
Oxygen therapy & 10 & 40.0 \\
Anticoagulant & 7 & 28.0 \\
Other treatment & 2 & 8.0 \\
\hline
\end{tabular}

${ }^{\star}$ Others: Antitussive agents, Progesterone tablets.

Total number of pre-natal consultations during the study period $(n=1197)$.

83 suspected cases based on presenting complaints were tested by PCR

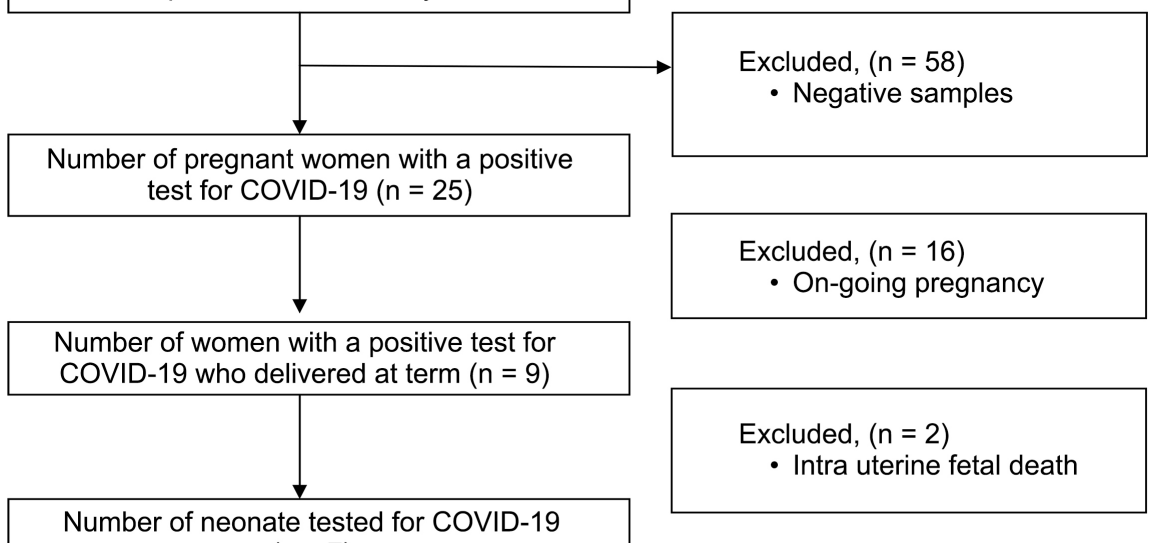

Figure 2. Description of study procedure and number of women and neonates followed-up. 
Table 3. Table showing maternal and foetal outcomes.

\begin{tabular}{|c|c|c|}
\hline Characteristics & Frequency $(n=25)$ & Percentage (\%) \\
\hline \multicolumn{3}{|l|}{ Pregnancy outcome } \\
\hline Abortion & 2 & 8.0 \\
\hline On-going pregnancy & 14 & 56.0 \\
\hline Delivery at term & 9 & 36.0 \\
\hline \multicolumn{3}{|l|}{ Mode of delivery $(n=9)$} \\
\hline Vaginal & 5 & 55.6 \\
\hline Caesarian section & 4 & 44.4 \\
\hline \multicolumn{3}{|l|}{ Indications for Caesarian section $(\mathrm{n}=4)$} \\
\hline Acute fotal distress syndrome & 3 & 75.0 \\
\hline Cervical dystocia and previous scar & 1 & 25.0 \\
\hline \multicolumn{3}{|l|}{ State of neonate at delivery $(n=9)$} \\
\hline Alive & 7 & 77.8 \\
\hline Dead & 2 & 22.2 \\
\hline Number of neonate PCR tested $(\mathrm{n}=7)$ & 7 & 100 \\
\hline PCR SARS-CoV-2 Negative & 7 & 100 \\
\hline
\end{tabular}

\section{Discussion}

Our study found out that, all seven babies born of COVID-19 positive pregnant women tested negative for the SARS-CoV-2 infection and showed no signs of abnormality. The median age of the pregnant women was 31 years. Most of them $(76 \%)$, consulted within 6 days of onset of symptoms suspicious of having COVID-19 after failure of the traditional herbal medicines to reduce their symptoms. This delay in consultation could be explained by the use of the traditional pharmacopoeia for symptoms initially considered mild [8].

During our study, we recorded ten cases (40\%) of Severe Acute Respiratory Distress Syndrome linked to COVID-19 pneumonia requiring transfer to intensive care. Due to physiological and immunological changes, pregnant women are more susceptible to severe pneumonia [9] [10]. This was noticed during the influenza pandemic in 1918 which caused a mortality rate among pregnant women of $37 \%$ against $2.6 \%$ in the general population [11]. Our findings are similar to those of Wong et al. [12] who found 33\% of severe forms in pregnant women with SARS and there are studies which have shown up to $79 \%$ sequence similarity between SARS-CoV-2 and SARS [13] [14]. We did not register any death in our series; but we remain vigilant because the evolution and prognosis of COVID-19 pneumonia could follow the same profile as SARS-CoV-1 and MERS-CoV. 
In general, the clinical characteristics are similar in pregnant or non-pregnant women [15]. The most frequent symptoms at the start of COVID-19 pneumonia in our series were fever, cough, and asthenia which have been found in similar proportions in other published studies [16] [17]; while the least common symptoms were diarrhea, dizziness, anorexia, and chest pain.

All of our study participants received an anti-parasitic/antibiotic association treatment in accordance with the directives of the scientific council of the Ministry of Public Health of Cameroon [18]. Only ten women benefited from oxygen therapy who presented with severe respiratory distress (temperature $\geq 38^{\circ} \mathrm{C}$, respiratory rate $>30$ cycles/minute, respiratory distress, $\mathrm{SpO}_{2}<90 \%$ in ambient air, cyanosis) requiring permanent oxygen therapy to maintain their $\mathrm{SpO}_{2}>92 \%$.

Five $(55.6 \%)$ women delivered vaginally, four underwent an emergency cesarean and two had an abortion. The indications for cesarean sections were fetal distress and cervical dystocia in a patient with a past history of cesarean section. Two Chinese studies show that their route of delivery was caesarean in $100 \%$ and $76.9 \%$ respectively [7] [19]. Two (22.24\%) of the five vaginal deliveries occurred while the patients were under investigation for their pneumonia; the other deliveries were performed after the patients were cured of their pneumonia with COVID-19. We do not have the data necessary to assess the risks to the newborn from per vaginal delivery to those of cesarean section. Seven (77.8\%) fetuses were alive with an APGAR score greater than 7 in the first minute and we have two neonatal deaths. It was difficult to formally incriminate the SARS-CoV-2 virus, but it is possible that these complications reflect poor tolerance to hypoxia [19]. Nasopharyngeal swabs were taken from newborns no later than 48 hours after birth and all were negative; no newborn presented with symptoms during admission and required pediatric care. Huijun et al. [7] did not find neonatal complications in his series of nine births, all performed by cesarean section.

We did not find any association between the occurrence of severe COVID-19 pneumonia and the unfavorable prognosis of pregnancy in our series. This could be justified by the limited sample size and also by the fact that the majority of pregnancies are in progress. The patients were recruited every trimester of pregnancy and the causal relationship of COVID-19 on the unfavorable outcome of the pregnancy seems difficult to establish.

\section{Limitations}

The small size of our sample does not allow definitive conclusions to be drawn. The follow-up of the remaining pregnant women will allow us to propose new results for the next work.

\section{Conclusion}

The maternal-fetal consequences of COVID-19 are not very frequent if we have a close follow-up. The data of our series follows the international pattern, so we 
recommend to the practitioners, to continue screening on a large scale and in a systematic way.

\section{Acknowledgments}

The authors would like to thank the patients for having given their consent to participate in the study carried out as well as the administration of the central hospital and its staff who mobilized in the care of these patients.

\section{Declarations}

\section{Ethics Approval and Consent to Participate}

The ethical approval has been obtained from the ethics committee of the Central Hospital of Yaounde. We didn't need to obtain informed consent from a parent or guardian as our patients were all over the age of 18 .

\section{Availability of Data and Materials}

The datasets generated and/or analyzed during the current study are not accessible to the public because they are the subject of ongoing research, but are available from the corresponding author on reasonable request.

These patients have not been reported in any other submission by me or anyone else.

\section{Author's Contributions}

MN Dingom and ES originated and designed the study.

$\mathrm{MN}, \mathrm{AA}, \mathrm{YW}$ contributed to data collection and clean. AN and JK conducted data analysis. MD, ES, GN, AN interpreted the findings and drafted the manuscript.

MN, ES, FE, AA, YW, JN, PS, CS, BW, GL, JB, FE, FF, GN, JF, RT, PO, PF, $\mathrm{RM}$ took care of the patients during their hospitalization.

All authors have read and approved the manuscript.

\section{Conflicts of Interest}

The authors declare no conflicts of interest regarding the publication of this paper.

\section{References}

[1] Zhu, N., Zhang, D., Wang, W., Li, X., Yang, B., Song, J., et al. (2020) A Novel Coronavirus from Patients with Pneumonia in China, 2019. New England Journal of Medicine, 382, 727-733. https://doi.org/10.1056/NEJMoa2001017

[2] World Health Organisation (n.d.) Coronavirus Diseases (COVID-19). Situation Report-179, 18.

[3] Ksiazek, T.G., Erdman, D., Goldsmith, C.S., Zaki, S.R., Peret, T., Emery, S., et al. (2003) A Novel Coronavirus Associated with Severe Acute Respiratory Syndrome. New England Journal of Medicine, 348, 1953-1966. https://doi.org/10.1056/NEJMoa030781 
[4] Zaki, A.M., van Boheemen, S., et al. (2012) Isolation of a Novel Coronavirus from a Man with Pneumonia in Saudi Arabia. New England Journal of Medicine, 367, 1814-1820. https://pubmed.ncbi.nlm.nih.gov/23075143

[5] Lescure, F.-X., et al. (2020) Clinical and Virological Data of the First Cases of COVID-19 in Europe: A Case Series. The Lancet Infectious Diseases, 20, 697-706. https://pubmed.ncbi.nlm.nih.gov/32224310

[6] Yu, N., Li, W., Kang, Q., Xiong, Z., Wang, S., Lin, X., et al. (2020) Clinical Features and Obstetric and Neonatal Outcomes of Pregnant Patients with COVID-19 in Wuhan, China: A Retrospective, Single-Centre, Descriptive Study. The Lancet Infectious Diseases, 20, 559-564. https://doi.org/10.1016/S1473-3099(20)30176-6

[7] Chen, H., Guo, J., Wang, C., Luo, F., Yu, X., Zhang, W., et al. (2020) Clinical Characteristics and Intrauterine Vertical Transmission Potential of COVID-19 Infection in Nine Pregnant Women: A Retrospective Review of Medical Records. The Lancet (London, England), 395, 809-815. https://doi.org/10.1016/S0140-6736(20)30360-3

[8] Traore, M. (n.d.) Le Recours à la Pharmacopée Traditionnelle Africaine dans le nouveau Millenaire: Cas de femmes Herboristes de Bamako.

[9] Jamieson, D.J., Theiler, R.N. and Rasmussen, S.A. (2006) Emerging Infections and Pregnancy. Emerging Infectious Diseases, 12, 1638-1643. https://doi.org/10.3201/eid1211.060152

[10] Warning, J.C., McCracken, S.A. and Morris, J.M. (2011) A Balancing Act: Mechanisms by Which the Fetus Avoids Rejection by the Maternal Immune System. Reproduction (Cambridge, England), 141, 715-724. https://doi.org/10.1530/REP-10-0360

[11] Gottfredsson, M. (2008) The Spanish Flu in Iceland 1918. Lessons in Medicine and History. Laeknabladid, 94, 737-745.

[12] Wong, G., Liu, W., Liu, Y., Zhou, B., Bi, Y. and Gao, G.F. (2015) MERS, SARS, and Ebola: The Role of Super-Spreaders in Infectious Disease. Cell Host Microbe, 18, 398-401. https://doi.org/10.1016/j.chom.2015.09.013

[13] Schoeman, D. and Fielding, B.C. (2019) Coronavirus Envelope Protein: Current Knowledge. Virology Journal, 16, 69. https://doi.org/10.1186/s12985-019-1182-0

[14] Zhang, Y.-Z. and Holmes, E.C. (2020) A Genomic Perspective on the Origin and Emergence of SARS-CoV-2. Cell, 181, 223-227.

https://doi.org/10.1016/j.cell.2020.03.035

[15] Huang, C., Wang, Y., Li, X., Ren, L., Zhao, J., Hu, Y., et al. (2020) Clinical Features of Patients Infected with 2019 Novel Coronavirus in Wuhan, China. The Lancet, 395, 497-506. https://doi.org/10.1016/S0140-6736(20)30183-5

[16] Wu, C., Chen, X., Cai, Y., Xia, J., Zhou, X., Xu, S., et al. (2020) Risk Factors Associated with Acute Respiratory Distress Syndrome and Death in Patients with Coronavirus Disease 2019 Pneumonia in Wuhan, China. JAMA Internal Medicine, 180, 934-943. https://doi.org/10.1001/jamainternmed.2020.0994

[17] Zhou, F., Yu, T., Du, R., Fan, G., Liu, Y., Liu, Z., et al. (2020) Clinical Course and Risk Factors for Mortality of Adult Inpatients with COVID-19 in Wuhan, China: A Retrospective Cohort Study. The Lancet (London, England), 395, 1054-1062. https://doi.org/10.1016/S0140-6736(20)30566-3

[18] Gautret, P., et al. (2020) Clinical and Microbiological Effect of a Combination of Hydroxychloroquine and Azithromycin in 80 COVID-19 Patients with at Least a Six-Day Follow Up: A Pilot Observational Study. Travel Medicine and Infectious Disease, 34, Article ID: 101663. https://pubmed.ncbi.nlm.nih.gov/32289548 
[19] Liu, Y., Chen, H., Tang, K. and Guo, Y. (2020) Clinical Manifestations and Outcome of SARS-CoV-2 Infection during Pregnancy. Journal of Infection. https://doi.org/10.1016/j.jinf.2020.02.028 